\title{
Bioefficacy of Coragen against Shoot and Fruit Borer, Earias vittella (Fab.) in Okra
}

\author{
Sanjay Kumar ${ }^{1 *}$, Vimal Kumar Singh ${ }^{1}$, Arvind Kumar ${ }^{2}$ and Naveen Chandra ${ }^{3}$ \\ ${ }^{1}$ Krishi Vigyan Kendra, Ujhani, Badaun (U.P.), India \\ ${ }^{2}$ Krishi Vigyan Kendra, Moradabad (U.P.), India \\ ${ }^{3}$ Krishi Vigyan Kendra, Meerut (U.P.), India \\ *Corresponding author
}

\section{A B S T R A C T}

K e y w o r d s
Okra, Coragen,
Spinosad, Earias
vittella, Shoot and
fruit borer.

\section{Keywords}

Okra, Coragen Spinosad, Earia fruit borer.
A field experiment was conducted on Okra, Abelmoschus esculentus (L.) to evaluate some newer groups of insecticides against shoot and fruit borer, Earias vittella. The experiment was lay out in RBD with three replication comprising eight treatments i.e. Indoxacarb @75g a.i/ha, Coragen@30g a.i/ha, Spinosad@100g a.i/ha, Profenophos@750g a.i/ha, Thiodicarb@750g a.i/ha, Bacillus thuringiensis@75g a.i/ha, Azadirachtin@2.5lit /ha including untreated control were taken for compression. The Coragen treated plot recorded lowest mean fruit damage (6.29 and 6.83\%) followed by Spinosad (7.17 and 7.47\%) and in control (30.96 and 33.14\%). The maximum yield was recorded in treatment Coragen (118.02 and $117.10 \mathrm{q} / \mathrm{ha}$ ) followed by Spinosad (115.65 and $114.85 \mathrm{q} / \mathrm{ha})$ as compare to control (68.80 and $68.10 \mathrm{q} / \mathrm{ha})$ respectively during Kharif, 2012 and Kharif, 2013. The highest cost benefit ratio was obtained in Coragen treated plot (1:9.27) followed by Indoxacarb (1:8.08), Spinosad (1:7.89) respectively. The Coragen treatment was more effective to reduce the infestation of fruit as well as to provide highest okra yield.

\section{Introduction}

Okra (Abelmoschus esculentus L. Moench.) is one of the important malvaceaeous vegetable crops grown all over India and various tropical and subtropical parts of the world. It is called lady's finger in England, gumbo in the United States of America and Bhindi in India. India has area under okra 511 thousand ha and production 5849 thousand MT during 2015-16. About 13 insect pests have been recorded that are known to cause damage to okra (Mandal et al., 2006). Among all pests, shoot and fruit borer, Earias vittella (Fabricius) is the most destructive pest of okra as young larva bores into tender shoot in early vegetative growth of plants and grown up larva damages fruits resulting in serious loss in yield. It is one of the major limiting factors in the production of quality fruits of okra (Kharbade et al., 1998). The infestation to okra accounted for nearly $22.5 \%$ in Uttar Pradesh (Verma et al., 1985). Earias vittella causes considerable damage at vegetative and reproductive stages and acts as major constraints in the production of marketable fruits of okra.

\section{Materials and Methods}

The experiment was conducted during the Kharif seasons 2012 and 2013 at Students 
Instructional Farm, NDUA\&T, Kumarganj, Faizabad (UP).The seed of okra (varietyArka Anamika) was sown in the month of last week of June in both season and all the agronomic and cultural practices recommended for its cultivation were followed as per the requirement. The crop was sown in the plots of $4.5 \mathrm{~m} \mathrm{x} 3 \mathrm{~m}$ with row to row and plant to plant spacing $60 \mathrm{~cm} \mathrm{x} 45$ $\mathrm{cm}$ respectively. Eight treatments including untreated control were taken for experimentation. The experiment was lay out in RBD and replicated thrice comprising Indoxacarb@75g a.i/ha, Coragen@30g a.i/ha, Spinosad@100g a.i/ha, Profenophos@750g a.i/ha, Thiodicarb@750g a.i/ha, Bacillus thuringiensis@75g a.i/ha, Azadirachtin@2.5lit/ha. All the treatments were applied three times at fortnightly interval using knapsack sprayer. Observation on damage and healthy fruits were recorded separately at each picking to find out the per cent fruit damage. After the last picking, total of all pickings of individual plots produce were calculated to work out the yield of the treatment. Yield of green healthy fruits of each plot was converted into quintal per hectare. The economics of treatment was calculated in term of cost: benefit ratio on the basis of pooled data of fruit yield. All the data were subjected to standard statistical analysis after arc sine transformation to draw the conclusion. The per cent fruit damaged and cost: benefit ratio were calculated by using the following formulae.

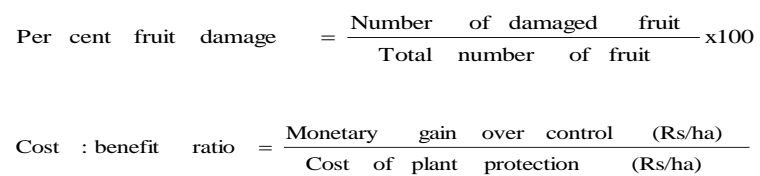

\section{Results and Discussion}

\section{Fruit damage}

The per cent damage fruit on number basis showed significant differences among all the treatments (Tables 1 and 2). Minimum fruit damage by Earias spp on okra was recorded in treatment (T2) coragen (5.50 and 6.38\%) followed by T3 (spinosad), T1 (indoxacarb), T4 (profenophos),T5 (thiodicarb),T6 Bt, T7 (Azadirachtin) in which (6.27 and 7.12), (7.15 and 7.80), (12.33 and 13.85), (12.68 and 14.00), (13.75 and 15.78), (15.75 and 16.14) per cent fruit damage respectively in compared to unprotected check (30.32 and 28.70) after Ist spraying at $7^{\text {th }}$ and $15^{\text {th }}$ days of treatments in Kharif, 2012 and the similar damage pattern was also recorded in Kharif, 2013. Data recorded at 7 th and 15 th days after IInd spray showed significant differences in all the treatment in compared to unprotected check in both Kharif seasons. Treatment (T2) was most effective $(6.78$ and $7.35 \%)$ followed by $\mathrm{T} 3>\mathrm{T} 1>\mathrm{T} 4>\mathrm{T} 5>\mathrm{T} 6>\mathrm{T} 7$ in which $(7.15$ and 7.95), (7.60 and 8.50), (12.50 and 15.50), (12.66 and 16.20), (15.25 and 17.25) and (15.80 and 17.50) per cent fruit damage were noticed during the Kharif, 2012 while (7.10 and 7.65), (7.25 and 8.15), (8.05 and 9.30), (12.85 and 16.12), (13.50 and 17.15), (15.75 and 17.50) and (16.30 and 18.50) per cent damaged fruit respectively during Kharif, 2013. After the III spray treatments T2 had the least fruit damage (5.60 and $6.12 \%)$ followed by T3, T1, T4, T5, T6, T7 in which (6.80 and 7.70), (7.12 and 8.00), (11.75 and 12.15), (12.05 and 13.50), (13.16 and 15.55), (14.00 and 17.75) and (31.60 and 30.50) per cent fruit damaged were recorded, respectively during Kharif, 2012. While in Kharif, 2013 it was T2> T3> T1> T4> T5> T6> T7 and T8 with respective fruit damage of (5.80 and 6.40), (7.12 and 7.90), (7.50 and 8.30), (11.09 and 13.77), (12.15 and 14.20), (13.25 and 16.25), (15.60 and 18.50) and (36.10 and 31.12) per cent, respectively.

The pooled mean per cent fruit infestation varied from 6.29 to 30.96 per cent. Coragen T2 was most effective $(6.29 \%)$ which was at par with treatment $\mathrm{T} 3$ spinosad $(7.17 \%)$ and $\mathrm{T} 1$ indoxacarb $(7.70 \%)$ followed by $\mathrm{T} 4$ 
profenophos (13.01\%), T5 thiodicarb $(13.52 \%), \quad \mathrm{T} 6 \quad \mathrm{Bt} \quad(15.12 \%)$ and $\mathrm{T} 7$ azadirachtin (16.16\%) as compared to control (30.96\%) respectively during Kharif 2012. In Kharif 2013, percent fruit infestation on number basis varied from 6.83 to 33.14 per cent. The minimum fruit damage of $(6.83 \%)$ was recorded in T2 coragen, while T3 spinosad $(7.47 \%)$ was second effective treatment followed by $\mathrm{T} 1$ indoxacarb (8.22\%), T4 profenophos (13.41\%), T5 thiodicarb (14.46\%), T6 Bt (15.90\%) and T7 azadirachtin (17.03\%) as compared to control 33.14 per cent fruit infestation.

The overall pooled mean of both Kharif seasons based on fruit damage showed the maximum fruit damage was recorded in treatment $\mathrm{T} 7$ azadirachtin $(16.60 \%)$ and minimum $(6.56 \%)$ in $\mathrm{T} 2$ coragen followed by T3 spinosad (7.32\%), T1 indoxacarb (7.96\%), T4 profenophos (13.21\%), T5 thiodicarb (13.99\%), T6 Bt (15.51\%) as compared to control $(32.05 \%)$. The coragen was most effective treatment followed by spinosad and indoxacarb and azadirachtin was least effective treatment.

These finding collaborated with the result of Sandip et al., (2009), Singh et al., (2010) and Chowdary, et al., (2010) who had reported that the Coragen (rynaxypyr) 20 SC @ $30 \mathrm{~g}$ a.i. /ha and Coragen 20 SC @ $20 \mathrm{~g}$ a.i. /ha were superior to check the fruit damage and higher okra yield followed by spinosad @ 56 g a.i/ha. Gosalwad and Kawathekar (2009) reported that the minimum fruit infestation was observed in spinosad 45\% SC @ $30 \mathrm{~g}$ a.i. /ha Shinde et al., (2011) noticed that Spinosad $0.005 \%$ and indoxacarb $0.01 \%$ were the most effective insecticides in controlling okra shoot and fruit borer. Highest yield of healthy okra fruit was observed in spinosad @0.005 percent. Singh et al., (2010) reported that the Indoxacarb $14.5 \% \mathrm{SC}$ proved to be the most effective treatment against shoot and fruit borer, Earias vittella $\mathrm{F}$. in and it was at par with spinosad. Mandal et al., (2006) reported that the Bacillus thuringensis @ $500 \mathrm{~g} / \mathrm{ha}$ and found to be the least effective treatment while, the Meena et al., (2011) reported Azadirachtin (5m1/lit.) was least effective followed by NPV $(0.010 \%)$ and B.t. $(0.012 \%)$. The findings of these workers are also agreement with the present study.

\section{Fruit yield and cost benefit ratio}

All the treatment gave higher fruit yield over unprotected control during both the Kharif, seasons (Table 3). The coragen @ 30g a.i. / ha treated plot gave maximum fruit yield (118.02 and $117.10 \mathrm{q} / \mathrm{ha}$ ) respectively during Kharif 2012 and 2013 followed by T3> T1> T4>T5> T6> and T7 with respective fruit yields of (115.65 and114.85), (105.35 and 104.25), (84.20 and 82.80), (81.60 and 80.84), (77.24 and 76.10) and (73.74 and 72.90) q/ha respectively were significantly superior over unprotected check (68.80 and $68.10 \mathrm{q} / \mathrm{ha})$ during Kharif 2012 and 2013. Similar finding was also reported by Umrao et al., (2012), Shimoge and Vemuri (2014) who have reported that the indoxacarb was most effective among all the treatments evaluated against shoot and fruit borer on okra.

Net monitory return in different treatment varied from Rs. 3555 to 65715 per hectare (Table 3). The maximum profit of Rs. 65715/ ha was obtained from the plot treated with $\mathrm{T} 2$ coragen followed by T3 spinosad Rs. 61300, T1 indoxacarb Rs 47775, T4 profenophos Rs. 18585, T5 thiodicarb Rs. 14505, T6 Bt Rs. 9930 and least in T7 Azadirachtin Rs. 3555, respectively. The cost benefit ratio (CBR) in different treatment ranged 1:1.95 to 1:9.27, however the highest cost benefit ratio of 1:9.27 per rupee was obtained in T2 treatment followed by $\mathrm{T} 1(1: 8.08)>\mathrm{T} 3(1: 7.89)>\mathrm{T} 4$ $(1: 5.66)>\mathrm{T} 6(1: 4.12)>\mathrm{T} 5(1: 4.11)$ and $\mathrm{T} 7$ (1:1.95), respectively. 
Table.1 Effects of insecticide against Earias vittella based on per cent fruit damage on okra (Kharif, 2012)

\begin{tabular}{|c|c|c|c|c|c|c|c|c|c|c|}
\hline \multirow{3}{*}{ S.No } & \multirow{3}{*}{ Treatments } & \multirow{3}{*}{ a.i/ha } & \multirow{3}{*}{$\begin{array}{c}\text { Pre } \\
\text { Treatment }\end{array}$} & \multicolumn{7}{|c|}{ Per cent Fruit Damage DAS (Days After Spraying) } \\
\hline & & & & \multicolumn{2}{|c|}{ I $^{\text {st }}$ Spray } & \multicolumn{2}{|c|}{ II $^{\text {nd }}$ Spray } & \multicolumn{2}{|c|}{ III $^{\text {rd }}$ Spray } & \multirow[t]{2}{*}{ Mean } \\
\hline & & & & 7 DAS & 15 DAS & 7 DAS & 15 DAS & 7 DAS & 15 DAS & \\
\hline $\mathrm{T}_{1}$ & Indoxacarb $14.5 \mathrm{EC}$ & $75 \mathrm{~g}$ & $\begin{array}{c}21.77 \\
(27.76)\end{array}$ & $\begin{array}{c}7.15 \\
(15.51)\end{array}$ & $\begin{array}{c}7.80 \\
(16.21)\end{array}$ & $\begin{array}{c}7.60 \\
(16.00)\end{array}$ & $\begin{array}{c}8.50 \\
(16.94)\end{array}$ & $\begin{array}{c}7.12 \\
(15.47)\end{array}$ & $\begin{array}{c}8.00 \\
(16.43)\end{array}$ & $\begin{array}{c}7.70 \\
(16.87)\end{array}$ \\
\hline $\mathrm{T}_{2}$ & Coragen 18.5 SC & $30 \mathrm{~g}$ & $\begin{array}{c}19.75 \\
(26.34)\end{array}$ & $\begin{array}{c}5.50 \\
(13.56\end{array}$ & $\begin{array}{c}6.38 \\
(14.63)\end{array}$ & $\begin{array}{c}6.78 \\
(15.09)\end{array}$ & $\begin{array}{c}7.35 \\
(15.73)\end{array}$ & $\begin{array}{c}5.60 \\
(13.69)\end{array}$ & $\begin{array}{c}6.12 \\
(14.32)\end{array}$ & $\begin{array}{c}6.29 \\
(15.89)\end{array}$ \\
\hline $\mathrm{T}_{3}$ & Spinosad $45 \mathrm{SC}$ & $100 \mathrm{~g}$ & $\begin{array}{c}25.94 \\
(30.61)\end{array}$ & $\begin{array}{c}6.27 \\
(14.50)\end{array}$ & $\begin{array}{c}7.12 \\
(15.47)\end{array}$ & $\begin{array}{c}7.15 \\
(15.51)\end{array}$ & $\begin{array}{c}7.95 \\
(16.38)\end{array}$ & $\begin{array}{c}6.80 \\
(15.11)\end{array}$ & $\begin{array}{c}7.70 \\
(16.11)\end{array}$ & $\begin{array}{c}7.17 \\
(16.46)\end{array}$ \\
\hline $\mathrm{T}_{4}$ & Profenophos 50 EC & $750 \mathrm{~g}$ & $\begin{array}{c}21.54 \\
(27.60)\end{array}$ & $\begin{array}{c}12.33 \\
(20.56)\end{array}$ & $\begin{array}{c}13.85 \\
(21.85)\end{array}$ & $\begin{array}{c}12.50 \\
(20.70)\end{array}$ & $\begin{array}{c}15.50 \\
(23.18)\end{array}$ & $\begin{array}{c}11.75 \\
(20.05)\end{array}$ & $\begin{array}{c}12.15 \\
(20.39)\end{array}$ & $\begin{array}{c}13.01 \\
(19.84)\end{array}$ \\
\hline $\mathrm{T}_{5}$ & Thiodicarb $75 \mathrm{WP}$ & $750 \mathrm{~g}$ & $\begin{array}{c}22.70 \\
(28.44)\end{array}$ & $\begin{array}{c}12.68 \\
(20.86)\end{array}$ & $\begin{array}{c}14.00 \\
(21.97)\end{array}$ & $\begin{array}{c}12.66 \\
(20.84)\end{array}$ & $\begin{array}{c}16.20 \\
(23.73)\end{array}$ & $\begin{array}{c}12.05 \\
(20.40)\end{array}$ & $\begin{array}{c}13.50 \\
(21.56)\end{array}$ & $\begin{array}{r}13.52 \\
(20.09)\end{array}$ \\
\hline $\mathrm{T}_{6}$ & Bt $5 \%$ & $75 \mathrm{~g}$ & $\begin{array}{c}23.95 \\
(29.27)\end{array}$ & $\begin{array}{c}13.75 \\
(21.76)\end{array}$ & $\begin{array}{c}15.78 \\
(23.40)\end{array}$ & $\begin{array}{c}15.25 \\
(22.98)\end{array}$ & $\begin{array}{c}17.25 \\
(24.54)\end{array}$ & $\begin{array}{c}13.16 \\
(21.27)\end{array}$ & $\begin{array}{c}15.55 \\
(23.22)\end{array}$ & $\begin{array}{c}15.12 \\
(21.34)\end{array}$ \\
\hline $\mathrm{T}_{7}$ & Azadirachtin $0.03 \%$ & 2.5lit & $\begin{array}{c}26.36 \\
(30.89)\end{array}$ & $\begin{array}{c}15.75 \\
(23.38)\end{array}$ & $\begin{array}{c}16.14 \\
(23.69)\end{array}$ & $\begin{array}{c}15.80 \\
(23.42)\end{array}$ & $\begin{array}{c}17.50 \\
(24.73)\end{array}$ & $\begin{array}{c}14.00 \\
(21.97)\end{array}$ & $\begin{array}{c}17.75 \\
(24.92)\end{array}$ & $\begin{array}{c}16.16 \\
(22.87)\end{array}$ \\
\hline $\mathrm{T}_{8}$ & Untreated Control & - & $\begin{array}{c}28.06 \\
(31.90)\end{array}$ & $\begin{array}{c}30.32 \\
(33.40)\end{array}$ & $\begin{array}{c}28.70 \\
(32.39)\end{array}$ & $\begin{array}{c}30.15 \\
(30.30)\end{array}$ & $\begin{array}{c}34.50 \\
(35.97)\end{array}$ & $\begin{array}{c}31.60 \\
(34.20\end{array}$ & $\begin{array}{c}30.50 \\
(33.52)\end{array}$ & $\begin{array}{r}30.96 \\
(32.55)\end{array}$ \\
\hline & SEm \pm & & 1.40 & 0.26 & 0.18 & 0.39 & 0.31 & 0.27 & 0.23 & 0.27 \\
\hline & $\mathrm{CD}$ at $5 \%$ & & $\mathrm{NS}$ & 0.80 & 0.54 & 1.17 & 0.95 & 0.82 & 0.70 & 0.83 \\
\hline
\end{tabular}

*The data in parenthesis is arc sine transformation value

NS- Non significant 
Table.2 Effects of insecticide against Earias vittella based on per cent fruit damage on okra (Kharif, 2013)

\begin{tabular}{|c|c|c|c|c|c|c|c|c|c|c|}
\hline \multirow{3}{*}{ S.No } & \multirow{3}{*}{ Treatments } & \multirow{3}{*}{ a.i/ha } & \multirow{3}{*}{$\begin{array}{c}\text { Pre } \\
\text { Treatment }\end{array}$} & \multicolumn{7}{|c|}{ Per cent Fruit Damage DAS (Days After Spraying) } \\
\hline & & & & \multicolumn{2}{|c|}{$\mathbf{I}^{\text {st }}$ Spray } & \multicolumn{2}{|c|}{ IInd Spray } & \multicolumn{2}{|c|}{ III $^{\text {rd }}$ Spray } & \multirow[t]{2}{*}{ Mean } \\
\hline & & & & 7 DAS & 15 DAS & 7 DAS & 15 DAS & 7 DAS & 15 DAS & \\
\hline $\mathrm{T}_{1}$ & Indoxacarb $14.5 \mathrm{EC}$ & $75 \mathrm{~g}$ & $\begin{array}{c}21.77 \\
(27.81)\end{array}$ & $\begin{array}{c}7.65 \\
(16.05)\end{array}$ & $\begin{array}{c}8.50 \\
(16.94)\end{array}$ & $\begin{array}{c}8.05 \\
(16.48)\end{array}$ & $\begin{array}{c}9.30 \\
(17.75)\end{array}$ & $\begin{array}{c}7.50 \\
(15.89)\end{array}$ & $\begin{array}{c}8.30 \\
(16.74)\end{array}$ & $\begin{array}{c}8.22 \\
(16.64)\end{array}$ \\
\hline $\mathrm{T}_{2}$ & Coragen 18.5 SC & $30 \mathrm{~g}$ & $\begin{array}{c}18.68 \\
(25.56)\end{array}$ & $\begin{array}{c}6.80 \\
(15.11)\end{array}$ & $\begin{array}{c}7.25 \\
(15.62)\end{array}$ & $\begin{array}{c}7.10 \\
(15.45)\end{array}$ & $\begin{array}{c}7.65 \\
(16.05)\end{array}$ & $\begin{array}{c}5.80 \\
(13.93)\end{array}$ & $\begin{array}{c}6.40 \\
(14.65)\end{array}$ & $\begin{array}{c}6.83 \\
(15.14)\end{array}$ \\
\hline $\mathrm{T}_{3}$ & Spinosad 45 SC & $100 \mathrm{~g}$ & $\begin{array}{c}23.61 \\
(29.06)\end{array}$ & $\begin{array}{c}7.10 \\
(15.45)\end{array}$ & $\begin{array}{c}7.30 \\
(15.67)\end{array}$ & $\begin{array}{c}7.25 \\
(15.62)\end{array}$ & $\begin{array}{c}8.15 \\
(16.59)\end{array}$ & $\begin{array}{c}7.12 \\
(15.48)\end{array}$ & $\begin{array}{c}7.90 \\
(16.32)\end{array}$ & $\begin{array}{c}7.47 \\
(15.86)\end{array}$ \\
\hline $\mathrm{T}_{4}$ & Profenophos 50 EC & $750 \mathrm{~g}$ & $\begin{array}{c}22.87 \\
(28.55)\end{array}$ & $\begin{array}{c}12.60 \\
(20.79)\end{array}$ & $\begin{array}{c}14.00 \\
(21.97)\end{array}$ & $\begin{array}{c}12.85 \\
(21.01)\end{array}$ & $\begin{array}{c}16.12 \\
(23.67)\end{array}$ & $\begin{array}{c}11.09 \\
(19.43)\end{array}$ & $\begin{array}{c}13.77 \\
(21.78)\end{array}$ & $\begin{array}{c}13.41 \\
(21.44)\end{array}$ \\
\hline $\mathrm{T}_{5}$ & Thiodicarb 75 WP & $750 \mathrm{~g}$ & $\begin{array}{c}20.23 \\
(26.72)\end{array}$ & $\begin{array}{c}14.50 \\
(22.38)\end{array}$ & $\begin{array}{c}15.24 \\
(22.98)\end{array}$ & $\begin{array}{c}13.50 \\
(21.55)\end{array}$ & $\begin{array}{c}17.15 \\
(24.46)\end{array}$ & $\begin{array}{c}12.15 \\
(20.39)\end{array}$ & $\begin{array}{c}14.20 \\
(22.14)\end{array}$ & $\begin{array}{c}14.46 \\
(22.32)\end{array}$ \\
\hline $\mathrm{T}_{6}$ & Bt $5 \%$ & $75 \mathrm{~g}$ & $\begin{array}{c}19.28 \\
(25.95)\end{array}$ & $\begin{array}{c}15.80 \\
(23.42)\end{array}$ & $\begin{array}{c}16.85 \\
(24.23)\end{array}$ & $\begin{array}{c}15.75 \\
(23.38)\end{array}$ & $\begin{array}{c}17.50 \\
(24.73)\end{array}$ & $\begin{array}{c}13.25 \\
(21.35)\end{array}$ & $\begin{array}{c}16.25 \\
(23.77)\end{array}$ & $\begin{array}{c}15.90 \\
(23.48)\end{array}$ \\
\hline $\mathrm{T}_{7}$ & Azadirachtin $0.03 \%$ & 2.5lit & $\begin{array}{c}24.21 \\
(29.47)\end{array}$ & $\begin{array}{c}16.00 \\
(23.58)\end{array}$ & $\begin{array}{c}17.25 \\
(24.54)\end{array}$ & $\begin{array}{c}16.30 \\
(23.81)\end{array}$ & $\begin{array}{c}18.50 \\
(25.47)\end{array}$ & $\begin{array}{c}15.60 \\
(23.26)\end{array}$ & $\begin{array}{c}18.50 \\
(25.47)\end{array}$ & $\begin{array}{c}17.03 \\
(24.36)\end{array}$ \\
\hline $\mathrm{T}_{8}$ & Untreated Control & - & $\begin{array}{c}23.39 \\
(28.92)\end{array}$ & $\begin{array}{c}30.50 \\
(33.51)\end{array}$ & $\begin{array}{c}28.50 \\
(32.26)\end{array}$ & $\begin{array}{c}34.50 \\
(35.96)\end{array}$ & $\begin{array}{c}38.10 \\
(38.11)\end{array}$ & $\begin{array}{c}36.10 \\
(36.92)\end{array}$ & $\begin{array}{c}31.12 \\
(33.90)\end{array}$ & $\begin{array}{c}33.14 \\
(35.11)\end{array}$ \\
\hline & $\mathrm{SEm} \pm$ & & 1.03 & 0.36 & 0.35 & 0.24 & 0.31 & 0.35 & 0.36 & 0.33 \\
\hline & $\mathrm{CD}$ at $5 \%$ & & NS & 1.10 & 1.06 & 0.74 & 0.94 & 1.08 & 1.08 & 1.00 \\
\hline
\end{tabular}

*The data in parenthesis is arc sine transformation value

NS- Non significant 
Table.3 Economics of treatments for management of fruit borer in okra during Kharif, 2012 and 2013

\begin{tabular}{|c|c|c|c|c|c|c|c|c|c|c|c|}
\hline Treatment & $\begin{array}{c}\text { Dose } \\
\text { a.i/ha } \\
\text { (g) }\end{array}$ & $\begin{array}{l}\text { Quantity } \\
\text { required/ } \\
\text { ha }\end{array}$ & $\begin{array}{c}\text { Total } \\
\text { treatment } \\
\text { quantity }\end{array}$ & $\begin{array}{c}\text { Cost of } \\
\text { insecticide } \\
\text { (Rs/lit) }\end{array}$ & $\begin{array}{c}\text { Cost of } \\
\text { treatment } \\
\text { (Rs) }\end{array}$ & $\begin{array}{l}\text { Yield } \\
\text { (q/ha) }\end{array}$ & $\begin{array}{c}\text { Yield } \\
\text { saved } \\
\text { over } \\
\text { control } \\
\text { (q/ha) }\end{array}$ & $\begin{array}{c}\text { Value } \\
\text { of saved } \\
\text { yield } \\
\text { (Rs/ha) }\end{array}$ & $\begin{array}{c}\text { Gross } \\
\text { income } \\
\text { (Rs/ha) }\end{array}$ & $\begin{array}{c}\text { Net } \\
\text { income } \\
\text { (Rs/ha) }\end{array}$ & $\begin{array}{c}\text { C:B } \\
\text { Ratio }\end{array}$ \\
\hline $\begin{array}{l}\text { Indoxacarb } \\
14.5 \mathrm{EC}\end{array}$ & 75 & $500 \mathrm{ml}$ & $1.50 \mathrm{lit}$ & 3400 & 6750 & 104.80 & 36.35 & 54525 & 157200 & 47775 & $1: 8.08$ \\
\hline $\begin{array}{l}\text { Coragen } \\
18.5 \% \mathrm{SC}\end{array}$ & 30 & $150 \mathrm{ml}$ & 0.45 lit & 14000 & 7950 & 117.56 & 49.11 & 73665 & 176340 & 65715 & $1: 9.27$ \\
\hline $\begin{array}{l}\text { Spinosad } 45 \% \\
\mathrm{SC}\end{array}$ & 100 & $166 \mathrm{ml}$ & 0.50 lit & 14500 & 8900 & 115.25 & 46.80 & 70200 & 172875 & 61300 & $1: 7.89$ \\
\hline $\begin{array}{l}\text { Profenophos } \\
50 \% \text { EC }\end{array}$ & 750 & 1.50 lit & 4.50 lit & 520 & 3990 & 83.50 & 15.05 & 22575 & 125250 & 18585 & $1: 5.66$ \\
\hline $\begin{array}{l}\text { Thiodicarb } \\
75 \% \mathrm{WP}\end{array}$ & 750 & $1.00 \mathrm{Kg}$ & $3.00 \mathrm{Kg}$ & 1000 & 4650 & 81.22 & 12.77 & 19155 & 121830 & 14505 & $1: 4.12$ \\
\hline Bt $5 \%$ & 75 & $1.50 \mathrm{Kg}$ & $4.50 \mathrm{Kg}$ & 300 & 3000 & 76.67 & 8.22 & 12330 & 115005 & 9330 & $1: 4.11$ \\
\hline $\begin{array}{l}\text { Azadirachtin } \\
0.03 \%\end{array}$ & $2.5 \mathrm{lit} / \mathrm{ha}$ & 2.50 lit & 7.50 lit & 280 & 3750 & 73.32 & 4.87 & 7305 & 109980 & 3555 & $1: 1.95$ \\
\hline $\begin{array}{l}\text { Untreated } \\
\text { Control }\end{array}$ & - & - & - & - & - & 68.45 & - & - & 102675 & - & - \\
\hline
\end{tabular}

Cost of treatment includes labour, rent of sprayer and insecticide.

Labour charge= Rs.100/ labour, Sprayer rent=Rs. 25/day, Market price: okra= Rs. 15/Kg 
The T2 coragen treatment was more effective to reduce the shoot and fruit infestation as well as to provide highest okra yield. The present observations are conformity with Kumar et al., (2013) and Bansode et al., (2015) who has reported that indoxacarb 14.5 SC @ 0.5 lit/ha minimized the shoot and fruit damage of $1.6 \%$ and $3.3 \%$, respectively against the pest as well as gave maximum marketable yield.

\section{Acknowledgements}

The authors are thankful to the Director of Research, Narendra Dev University of Ag. \& Tech., Kumargang, Faizabad and Head, Department of Entomology, College of Agriculture for the unstinted support and other research facilities provided to carry out the investigation.

\section{References}

Bansode, A. G., Patil, C. S. and Jadhav, S. S. 2015. Efficacy of insecticides against shoot and fruit borer, Earias vittella F. infesting okra. Pest Management in Horticultural Ecosystems, 21(1): 106109.

Chowdary, L. R., Bheemanna, M., Kumar, L. R. 2010. Field efficacy of rynaxypyr (coragen) $20 \mathrm{SC}$ against fruit and shoot borer, Earias vittella (Fab.) in okra. International J. Plant Protection, 3(20):
316-318.

Kharbade, S.B., Chandel, A.G. and Dethe, M.D. 1998. Bioeffecacy of Bacillus thuringiensis (Berliner) varietal products against E. vittella (Fab.) on okra. Pestology, 22(11):8-10.

Mandal, S.K., Sah, S.B; Gupta, S.C. 2006. Screening of okra cultivars against Earias vitella. Ann. Pl. Protec. Sci., 14(2): 471-472.

Sandip, Patra Chatterjee, M. L. and Arunava, Samanta. 2009. Field efficacy of insecticides for management of Earias vittella in okra. Ann. Plant Protection Sciences, 17 (2): 471-472.

Shimoge, D., and Vemuri, S.B. 2014. Bioefficacy and dissipation of newer molecules against pests of okra. Indian Journal of Advances in Plant Research (1): 56-63.

Shinde, S.T., Shetgar, S.S and Badgujar, A.G. 2011. Bio-efficacy of different insecticides against major pest of okra. J. Entomological Research, 35(2): 133137.

Singh, S.S., Rai, M. K and Singh, V.B. 2010. Bio-efficacy of bio-rational insecticides and B.t. based bio-insecticides against shoot and fruit borer, Earias vittella in okra. Veg. Sci., 37(2): 171-174.

Verma, G. S., 1985. Response of chemicals to control spotted bollworm in okra. Ind. Jour. Entomology, 47(3): 357-358.

\section{How to cite this article:}

Sanjay Kumar, Vimal Kumar Singh, Arvind Kumar and Naveen Chandra. 2017. Bioefficacy of Coragen against Shoot and Fruit Borer, Earias vittella (Fab.) in Okra. Int.J.Curr.Microbiol.App.Sci. 6(10): 1021-1027. doi: https://doi.org/10.20546/ijcmas.2017.610.123 